\title{
Non-oestrogenic modalities to reverse urogenital aging
}

\author{
Simone Garzon ${ }^{1}$, Vasso Apostolopoulos ${ }^{2}$, Lily Stojanovska ${ }^{2,3}$, Federico Ferrari ${ }^{4}$, Begum Aydogan Mathyk ${ }^{5}$, \\ Antonio Simone Laganà
}

${ }^{1}$ Department of Obstetrics and Gynaecology, AOUI Verona, University of Verona, Verona, Italy

${ }^{2}$ Institute for Health and Sport, Victoria University, Melbourne VIC, Australia

${ }^{3}$ Department of Nutrition and Health, College of Medicine and Health Sciences, United Arab Emirates University, Al Ain,

United Arab Emirates

${ }^{4}$ Department of Obstetrics and Gynaecology, Spedali Civili of Brescia, Brescia, Italy

${ }^{5} \mathrm{HCA}$ Healthcare/University of South Florida Morsani College of Medicine GME Consortium, Brandon Regional Hospital, Brandon, FL, USA

${ }^{6}$ Department of Obstetrics and Gynaecology, "Filippo Del Ponte” Hospital, University of Insubria, Varese, Italy

\begin{abstract}
Urogenital aging is a common process affecting all women in the post-menopausal period of their life, and it is substantially due to oestrogen deprivation after ovarian function cessation. These changes can lead to a progressive, chronic, and complex association of symptoms identified as the genitourinary syndrome of menopause, which has a significant impact on quality of life. Genitourinary syndrome and urogenital aging do not resolve spontaneously and usually recur when treatment is stopped. Therefore, appropriate long-term management is of paramount importance, and local oestrogen is the most effective treatment to reverse urogenital aging and to improve symptoms of genitourinary syndrome as replacement therapy. In some women, topical oestrogen may be inconvenient, it may not achieve complete response, or it may be contra-indicated. Several non-hormonal therapies have been investigated, but few treatments have been reported as potentially able to reverse the urogenital aging process similarly to exogenous oestrogens. Laser seems the most promising, although further studies to define its safety and efficacy are mandatory. Vitamin D and E, and phytotherapy have returned conflicting results and require further confirmation. Lifestyle modifications, physiotherapy, and electrical stimulation represent inexpensive and applicable treatments that might slow urogenital aging. Among the hormonal nonoestrogenic therapies, the use of vaginal oxytocin and dehydroepiandrosterone have been found to be effective compared to placebo, as well as the use of oral ospemifene, which partially relieves vulvovaginal atrophy.
\end{abstract}

Key words: urogenital aging, genitourinary syndrome, non-hormonal therapy, vulvovaginal atrophy.

\section{Introduction}

The urogenital aging represents a common process affecting all women in the post-menopausal period of life. This process is caused by aging, but it is mainly determined by oestrogen deprivation following cessation of ovarian function. Due to the common embryological origin, both the lower urinary tract and the genitalia present oestrogen receptors that are dependent on oestrogen levels and actions for their trophism and physiology [1]. The hypoestrogenism of menopause explains the sudden and common changes of the genitalia and lower urinary tract. The nonkeratinized stratified squamous epithelium of vagina and urethra becomes pale, thin, and less regulated, with loss of glycogen production and subsequent reduction of lactobacillus and higher $\mathrm{pH}$, favouring colonization by enteric bacteria [2]. The reduced vascularization with the loss of dermal col- lagen, elastin, and hyaluronic acid determines changes of the vagina, vulva, bladder trigone, and urethra. The vagina becomes narrowed and shortened with the loss of rugae and compliance. The subcutaneous fat of the vulva decreases, the skin and mucosa become thinner, the tissues increase laxity, and sensitivity decreases [3]. The urethra and the trigone of the bladder lose their tone and trophism, there is a reduction of the urethra closure pressure with sphincter dysfunction and lower Valsalva leak-point pressure, and a decreased bladder sensory threshold when distended [4]. In addition, the levels of androgens contribute to the maintenance of genitourinary tissues expounding their role as a precursor of oestrogen biosynthesis and inducing changes in local androgen and oestrogen receptor expression, cell growth, mucin production, collagen turnover, increased perfusion, and neurotransmitter synthesis. In particular, according to recent data [5], elevated levels of tes- 


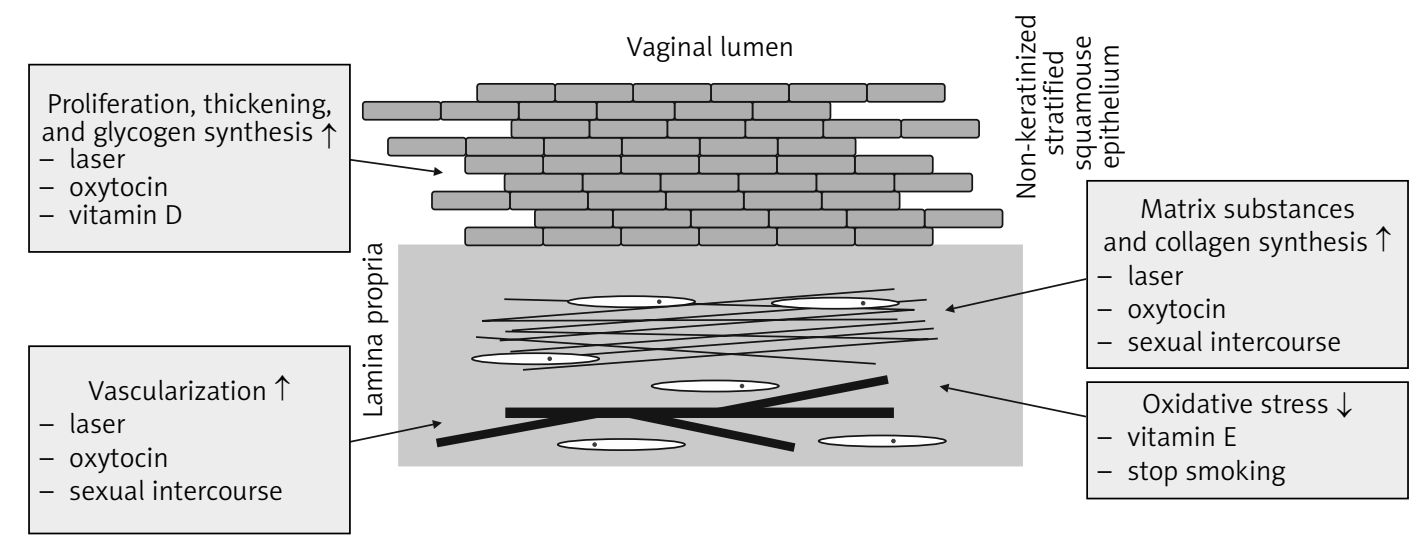

Fig. 1. Summary of the effects of laser, oxytocin, and vitamin D and $E$ on reversal of urogenital ageing

tosterone are related to severe psychological symptoms and total menopausal symptom scores; moreover, the decrease in progesterone levels is related to high psychological, urogenital, and total menopausal symptom scores; finally, elevated triglyceride levels are also related to the total severe symptom scores.

The complex pattern of anatomical and functional changes of urogenital aging can determine a progressive, chronic, and complex association of symptoms identified as the genitourinary syndrome of menopause (GSM) [6] (Fig. 1). This syndrome includes 2 categories of signs related to oestrogen deficiency, which are symptoms of vulvovaginal atrophy (VVA) and urinary symptoms. The changes associated with VVA, namely vaginal dryness, irritation, tenderness, and pain during intercourse, represent the most reported symptoms, which are associated with impairment of sexual satisfaction, intimacy, relationship with one's partner, and uncomfortable feelings with decreased quality of life [7, 8]. In this context, it is important to consider the differential diagnosis with other vulvar diseases, such as lichen planus and lichen sclerosus, which are known to be associated with altered genital self-image and sexual dysfunction [9]. Meanwhile, urinary urgency, frequency, urgent and stressed urinary incontinence, and recurrent urinary tract infections represent the main lower urinary tract dysfunction symptoms $[10,11]$. It is estimated that approximately $40-54 \%$ of postmenopausal women develop symptoms related to GSM, and considering the higher life expectancy, the prevalence is expected to rise [12].

Genitourinary syndrome does not resolve spontaneously, often progresses without treatment, and usually recurs when treatment is stopped [6]. On that basis, and considering the high prevalence and profound effect on the quality of life, appropriate management is of paramount importance. Treatment is often based on long-term therapy, aimed at reversing urogenital aging and easing symptoms, but is also based on the severity of the symptoms, patient preferences, and treatment effectiveness and safety $[13,14]$. In this context, the hormonal therapy, which is primarily based on local oestrogen, is the standard effective therapeutic approach for women with moderate-severe genitourinary symptoms or for those unresponsive to lubricants or moisturizers; in addition, recent data suggest that the use of levonorgestrel-releasing intrauterine system can concurrently improve urinary incontinence along with irritative and obstructive symptoms and thus positively affect the patients' quality of life in women with adenomyosis [15]. However, in women with previous history of oncologic hormone-dependent disease this approach might represents a relative contraindication; in fact, the decision for this treatment should be discussed and agreed with their oncologist $[16,17]$. The latter is particularly relevant for women receiving aromatase inhibitors with suppressed plasma levels of oestrogen after breast cancer, even though non-randomized evidence failed to show an increased risk of recurrence in these patients [18].

In women without adequate response to hormonal oestrogenic therapy, the use of non-oestrogen therapies to reverse urogenital aging as an alternative or additional approach is essential to achieve adequate improvement of genitourinary syndrome-related symptoms and to improve their quality of life. On that basis, this review provides a summary of available evidence on non-oestrogen modalities to reverse urogenital ageing, and to highlight the most effective treatments for the wide range of clinical symptoms of GSM.

\section{Material and methods}

A non-systematic review was conducted by searching the following databases: MEDLINE, EMBASE, Global Health, The Cochrane Library (Cochrane Database of Systematic Reviews, Cochrane Central Register of Controlled Trials, Cochrane Methodology Register), Health Technology Assessment Database and Web of Science, and research registers (such as www.clinicaltrials.gov). We used the medical subject heading (MeSH) term "Menopause" (MeSH Unique ID: D008593) in combination with "Therapy" (MeSH Unique ID: Q000628) 
and "Female Urogenital Diseases" (MeSH Unique ID: D052776). We selected papers written in English, with no time restrictions regarding the year of publication. Titles and/or abstracts of studies retrieved using the search strategy, and those from additional sources, were screened independently by 2 review authors to identify studies that potentially met the aims of this non-systematic review. The full text of these potentially eligible articles was retrieved and independently assessed for eligibility by another 2 review team members. Any disagreement between them over the eligibility of particular articles was resolved through discussion with a third (external) collaborator. Two authors independently extracted data from articles about study features and included populations, type of intervention (duration of therapy and drug posology), and outcomes. Any discrepancies were identified and resolved through discussion (with a third external collaborator where necessary). Due to the nature of the findings, we opted for a narrative synthesis of the results from selected articles.

\section{Limitations of oestrogen therapy}

Local oestrogen is the most effective treatment to reverse urogenital aging and to improve symptoms of genitourinary syndrome as replacement therapy [19-21]. Furthermore, of outmost importance, it is the role of local oestrogen in the improvement of urge incontinence symptoms by increasing urethral closure pressure and squamous metaplasia of the lower tract transitional epithelium [22]. On that basis, low-dose topical oestrogen is recommended and considered the therapeutic standard, respectively, in cases of moderate-severe symptoms or milder symptoms not responsive to lubricants and moisturizers and in the case of urge incontinence [23]. Following the initial minimal systemic absorption of oestrogen, given to the atrophic genital mucosa, this regimen is considered safe for the small increase of oestrogen circulating levels that does not require progesterone use to avoid unopposed endometrial exposure to oestrogen [23-25]. The rate of adverse effects, such as increased endometrial thickness and breast disorders, was not found to be significantly different between the different preparations or when compared to placebo [19].

Nevertheless, data regarding safety are limited. Randomized data regarding long-term exposure of endometrium to low doses of topical oestrogen are inconclusive [26], even though long-term observational data failed to demonstrate an increased risk of endometrial cancer. In general, the available data about adverse effects are not conclusive and further studies seem to be mandatory do draw firm conclusions [19, 23], where the most controversial issue regards the impact of severity of vaginal atrophy on systemic oestrogen absorption. However, accumulating observational data based on large observational studies are reassuring, in particular for low-dose oestrogen local application, and finally, the main limitations regard the inconvenience of use and the contraindications in the selected group of patients, as well as those after breast cancer [17, 23].

\section{Non-hormonal therapy}

Several non-hormonal therapeutic strategies for urogenital aging have been investigated. Moisturizers have been proposed as the first-line therapy for women with urogenital aging and mild symptoms [23], and they must be used on a regular basis to alleviate symptoms such as dryness and irritation. There are different moisturizing molecules, and some of them have been shown to have similar effectiveness in the management of symptoms related to the urogenital aging, such as dryness, irritation, and itching, as compared to oestrogens [27]. On the other hand, the use of lubricants could be episodical, alone or as supplemental treatment to moisturizers, and it has been suggested that they are best applied during intercourse to reduce pain, post-coital irritation, and the risk of traumatic lesions. Different lubricants based on water, artificial or plant oil, silicone, hybrids, or hyaluronic acid are also available. Of notable importance, a multicentre randomized trial focusing on the efficacy of vaginal hyaluronic acid in comparison to oestriol cream reported non-inferiority in reducing vaginal dryness with regards to the non-hormonal treatment [28]. However, although the lubricants are able to provide relief of symptoms, often the improvement is temporary, and frequent reapplications are required [13]. Of note, the lubricants and moisturizers do not reverse urogenital aging. Conversely, they are aimed to make up for the functional and anatomical deficiencies secondary to urogenital aging, improving coital comfort and maintaining vaginal secretions with their presence [29].

Although different non-hormonal therapies have been proposed for the management of urogenital aging-related symptoms, only some treatments have been reported as potentially able to reverse the urogenital aging process similarly to oestrogens.

\section{Laser treatment}

Laser therapy was introduced recently in the management of the urogenital aging as a treatment to reverse the aging process. Different studies investigated the application of lasers in symptomatic patients and reported an improvement of symptoms and of vaginal health index scores with patient satisfaction [30]. The 2 main types of lasers currently used for the treatment of GSM are the fractional microablative $\mathrm{CO}_{2}$ laser and the non-ablative photothermal erbium:YAG laser. The treatment with a $\mathrm{CO}_{2}$ laser was reported to improve 
different symptoms related to the vulvovaginal atrophy, such as dryness, itching, burning, dyspareunia, and dysuria, with an improvement of quality of sex life [31-33]. In a clinical randomized trial it was noted that the use of a $\mathrm{CO}_{2}$ laser alone or in combination with topical oestrogen provided improved dyspareunia, burning, and dryness when compared to exclusive oestrogen treatment [34]. Similarly, the erbium laser is able to stimulate the remodelling and synthesis of vaginal collagen that may repair and restore the pelvic floor function [35]. Accumulating evidence suggests that these results may be due, at least in part, to the reverse of urogenital aging. Laser treatment was related to an improvement of the vaginal mucosa vascularization, a stimulation of the new matrix substances, and collagen synthesis in the connective tissue, with a reported thickening of the vaginal epithelium, new papillae, and improved glycogen synthesis [36-38]. However, despite these promising results, the evidence is limited and is derived from studies evaluating short-term outcomes of efficacy and safety. In fact, the randomized evidence supporting the role of laser therapy against or combined with oestrogen topical therapy is scarce and only short-term results of up to 6 months have been reported [34, 39]. In regards to efficacy and safety, further controlled studies with long follow-up are required, and currently a multicentre trial is conducting a randomized study in patients affected by GSM among 2 types of laser treatment cohorts [40]. Moreover, the role of repeated treatment requires additional investigation [13, 41, 42]. This is mandatory following the 2018 Food and Drug Administration (FDA) statement regarding the safety and effectiveness of laser therapy for "vaginal rejuvenation", which has not been adequately evaluated and confirmed [43].

\section{Vitamin D and E}

Vitamin D has also been evaluated in the female reproductive physiology. Vitamin $D$ receptor was identified in the female reproductive organs, and the structure of vitamin D is analogous to the structure of progesterone as fat-soluble secosteroid hormone [44]. In addition, vitamin $D$ regulates the proliferation and promotes the differentiation of keratinocytes, such as those of the vaginal stratified squamous epithelium [45]. On that basis, it was proposed that vitamin D supplementation may promote vaginal epithelial maturation [46]. Available evidence from non-randomized clinical studies provides conflicting data regarding symptom improvement following vitamin D supplementation. There is limited support for the use of vitamin D for the treatment of urogenital aging, requiring further investigation, although an improved effect on epithelial cells was reported [46-49]. Similarly, limited non-randomized evidence supports the utility of vitamin E ap- plication as vaginal capsules to improve lubrication and urogenital aging symptoms. Anti-inflammatory and antioxidant functions of vitamin $\mathrm{E}$ might prevent damage by oxidative agents (Fig. 1) [23, 49, 50].

\section{Phytotheropy}

Forty to fifty per cent of women in western countries commonly use plant-based therapies as alternatives to hormone therapy. This alternative approach involves different plants, such as soy isoflavones, red clover, black cohosh, chasteberry, gingko biloba, chamomile, calendula flower, green tea, and other medicinal herbs [13]. A number of studies have investigated the effectiveness of these treatments in the management of menopausal symptoms, and although randomized controlled trials are available, the evidence is limited by small sample sizes, short follow-up, and inconsistence in data outcomes [51]. Furthermore, few studies have determined the effectiveness of these treatments on urogenital aging, reporting no evidence of changes of the vaginal epithelium, the maturation index, and urogenital symptoms with the exemption of vaginal dryness score [51-53].

\section{Lifestyle modifications, physiotherapy, and electrical stimulation}

Lifestyle plays a role in the prevention and reversion of urogenital aging. Sexual intercourse can play a role in the maintenance as well as the restoration of urogenital health and vaginal function, and in cases of vaginal narrowing or women without regular sexual intercourse, stretching of the vagina and the use of dilatators can be useful, although data are very limited [1, 23, 41]. Smoking cessation as well as weight loss represent further factors that can be taken into account. Smoking was associated with a higher risk of urinary urgency and frequency and accelerated urogenital aging [46, 54, 55]. Furthermore, weight loss (5-10\%) has been noted to reduce urinary incontinence and, for this reason, is highly recommended $[23,46]$. Meanwhile, cognitive behavioural therapy, physical activity, and acupuncture have all been reported to improve symptoms related to urogenital aging by evidence of various quality, although their effects are not clearly related to mechanisms that are able to reverse urogenital aging [46].

Lifestyle interventions can be combined with bladder training exercises, pelvic floor muscle training, and electrical stimulation [46]. Pelvic floor muscle and bladder training can slow or reverse urogenital aging via the improvement of pelvic organ support strength and intraurethral pressure during efforts, with improved tone and elasticity of tissues [56, 57]. Similarly, electrical stimulation by vaginal and anal electrodes has the po- 
tential to increase blood flow and trophism of the pelvic muscles, with a normalization of the lower urinary tract's reflex activity, and an improvement of symptoms in $30-50 \%$ of patients [58].

\section{Hormonal, non-oestrogenic therapies}

Among hormonal therapies, there are alternative non-oestrogenic medications that can be used to treat GSM. The 2 treatment options approved for vaginal use are oxytocin and dehydroepiandrosterone (DHEA), while ospemifene can be taken orally.

\section{Oxytocin}

Oxytocin is a neuropeptide hormone released by the posterior pituitary gland, and as such, an oxytocin gel was developed and investigated for its ability to treat urogenital aging [1]. Two randomized double-blinded placebo-controlled trials $[59,60]$ reported improved symptoms, which were related to improved normalized vaginal epithelium with a higher percentage of superficial mature cells and decreased $\mathrm{pH}$. Likewise, in a recent randomized controlled trial [61] it was clear that a higher rate of patients who used oxytocin had relief of dyspareunia and soreness compared to the control group. Interestingly, none of those studies reported effects on the circulating levels of oestradiol or on the endometrial thickness between groups $[59,61]$. Similarly, a double-blinded randomized placebo-controlled trial evaluated the effect of oxytocin on vaginal atrophy, but for a longer follow-up period (8 weeks), noting improved relief of symptoms, decreased $\mathrm{pH}$ of the vagina, and an improvement of the maturation index in the treatment group [62]. To date, no randomized controlled trials have been conducted that compare the effect of oxytocin with local oestrogen therapy.

Available evidence reports oxytocin involvement in cell proliferation signalling, and both in vivo and in vitro oxytocin have been found to increase cell proliferation. Oxytocin was related to an increased rate of wound healing, mucosal blood flow, as well as increased secretion of several growth factors in different cell types. This mechanism may explain the increased vaginal mucosal thickness and the improved vaginal symptoms in postmenopausal vaginal atrophy, supporting oxytocin as a molecule able to reverse urogenital aging (Fig. 1) [63].

\section{Dehydroepiandrosterone}

Vaginal and oral administration of DHEA is a valuable option for the treatment of dyspareunia in the context of GSM [64]; in fact, daily vaginal use of DHEA was approved by the FDA in 2016. The mechanism of action of DHEA on the vagina is due to the aromati- zation of androstenedione and testosterone locally to oestrone and oestradiol [65]. The dose efficacy of vaginal DHEA was demonstrated in 2 double-blind, placebo-controlled clinical trials of healthy postmenopausal women, who reported moderate to severe pain during sexual intercourse as their most relevant symptom of VVA. After 12 weeks, DHEA was shown to reduce the severity of pain experienced during sexual intercourse when compared to placebo $[66,67]$. Similarly, the safety of DHEA was demonstrate in a 52-week open-label trial, in which the most common adverse reactions were abnormal pap smear and increased vaginal discharge; the latter resulted mostly from the melting of the DHEA vehicle and the expected increase in vaginal secretion [68]. The levels of serum oestradiol and testosterone increased from baseline during DHEA treatment, but remained within normal postmenopausal ranges, and so far no effect on the endometrium has been reported $[69,70]$. However, no data beyond 12 weeks is available regarding potential risks associated with oestrogen or androgen therapy, such as breast cancer, ovarian cancer, and vascular ischaemic events or thrombosis. Nonetheless, for most women with symptomatic GSM who did not benefit from lubricants and moisturizers, a low-dose vaginal oestrogen therapy could be more appropriate than vaginal DHEA. In fact, there are more data and clinical experience with topical oestrogen, and its twice weekly dosing would be easier for most patients than the daily dosing of DHEA. Finally, to date, no randomized controlled trials have compared DHEA with other treatment options in postmenopausal women with VVA.

\section{Ospemifene}

Oral ospemifene is a selective oestrogen receptor modulator that acts as an oestrogen agonist in the vaginal tissue, and it does not have a clinically relevant oestrogenic effect on the breast and the endometrium [71]. Compared to placebo, ospemifene is effective in treating dyspareunia and vaginal dryness in the context of vulvovaginal atrophy, even though comparative studies with local oestrogen therapy are missing. Two randomized trials showed that daily oral administration of ospemifene for 12 weeks improved symptoms, although the benefits were modest $[72,73]$. In fact, the first randomized trial reported an objective evaluated reduction of dyspareunia of 53\% for ospemifene versus 39\% for placebo. Similarly, in the second trial the improvements in sexual pain, arousal, and desire domains in the female sexual arousal index (FSI) were greater for ospemifene. The main side effects of this treatment are hot flushes, reported with a prevalence of $7 \%$, while ospemifene appears safe regarding endometrium, without cases of atypical hyperplasia of endometrial cancer, even though it can increase slightly the thickening of the endometri- 
um $[73,74]$. No reports of thrombosis with ospemifene have been reported, but evidence is insufficient to depict this relatively rare complication [73].

\section{Conclusions}

Urogenital aging is a common condition of the menopause, and the main factor is oestrogen deprivation due to the hormone dependence for their normal trophism and physiology. This condition may be associated with genitourinary syndrome, which does not resolve spontaneously and often progresses, as well as to related urogenital aging. Only strategies able to stop or reverse urogenital aging may be effective in the treatment of the related syndrome and symptoms, such as oestrogen. Conversely, treatments aimed to make up for the functional and anatomical deficiencies secondary to the urogenital aging, such as lubricants and moisturizing, play a role in women with mild symptoms or as additional treatment. On that basis, hormone therapy is effective and recommended because it reverses urogenital aging. Similarly, any other methods able to reverse urogenital aging may provide further strategies to treat genitourinary syndrome, particularly in cases of insufficient or not applicable hormone therapy.

Although different non-hormonal modalities to reverse urogenital ageing have been investigated, the available strategies are supported by limited evidence. Therefore, further studies to define the safety and efficacy of these treatments are of paramount importance. Laser and oxytocin seem to be the most promising and require further long-term studies focused on the effectiveness and safety, with particular attention to longterm outcomes, the utility and modality of repeated treatment, and the adverse effects. Moreover, further investigation on the role of lifestyle modifications, interventions, physiotherapy, and electrical stimulation is mandatory, representing an inexpensive and applicable treatment able to at least slow urogenital aging.

Finally, it should be taken into account that early intervention in menopausal women is likely to be more effective than treatment after years of oestrogen deprivation and long-term urogenital aging. Therefore, clinicians should actively search for signs or symptoms of genitourinary syndrome and sexual problems, which are often not discussed, to start appropriate treatment as soon as possible.

\section{Acknowledgements}

The authors have no proprietary, financial, professional, or other personal interests of any nature in any product, service, or company. The authors alone are responsible for the content and writing of the paper. All the authors conform to the International Committee of
Medical Journal Editors (ICMJE) criteria for authorship, contributed to the intellectual content of the study, and gave approval for the final version of the article.

\section{Disclosure}

The authors report no conflict of interest.

\section{References}

1. Gandhi J, Chen A, Dagur G, et al. Genitourinary syndrome of menopause: an overview of clinical manifestations, pathophysiology, etiology, evaluation, and management. Am J Obstet Gynecol 2016; 215: 704-711.

2. Lüthje P, Hirschberg AL, Brauner A. Estrogenic action on innate defense mechanisms in the urinary tract. Maturitas 2014; 77: 32-36.

3. Basaran M, Kosif R, Bayar U, et al. Characteristics of external genitalia in pre- and postmenopausal women. Climacteric 2008; 11: 416-421.

4. Robinson D, Toozs-Hobson P, Cardozo L. The effect of hormones on the lower urinary tract. Menopause Int 2013; 19: 155-162.

5. Kaya C, Cengiz H, Yeşil A, et al. The relation among steroid hormone levels, lipid profile and menopausal symptom severity. J Psychosom Obstet Gynaecol 2017; 38: 284-291.

6. Portman DJ, Gass ML. Vulvovaginal Atrophy Terminology Consensus Conference Panel. Genitourinary syndrome of menopause: new terminology for vulvovaginal atrophy from the International Society for the Study of Women's Sexual Health and the North American Menopause Society. Maturitas 2014; 79: 349-354.

7. Nappi RE, Palacios S, Panay N, et al. Vulvar and vaginal atrophy in four European countries: evidence from the European REVIVE Survey. Climacteric 2015; 19: 188-197.

8. Simon JA, Kokot-Kierepa M, Goldstein J, et al. Vaginal health in the United States. Menopause 2013; 20: 1043-1048.

9. Yıldız Ş, Cengiz H, Kaya C, et al. Evaluation of genital self-image and sexual dysfunction in women with vulvar lichen planus or lichen sclerosus. J Psychosom Obstet Gynaecol 2020; 1-8.

10. Calleja-Agius J, Brincat MP. The urogenital system and the menopause. Climacteric 2015; 18(sup1): 18-22.

11. Robinson D, Cardozo LD. The role of estrogens in female lower urinary tract dysfunction. Urology 2003; 62: 45-51.

12. DiBonaventura $M$, Luo X, Moffatt $M$, et al. The Association Between Vulvovaginal Atrophy Symptoms and Quality of Life Among Postmenopausal Women in the United States and Western Europe. J Women Heal 2015; 24: 713-722.

13. Palacios S, Castelo-Branco C, Currie H, et al. Update on management of genitourinary syndrome of menopause: a practical guide. Maturitas 2015; 82: 308-313.

14. The North American Menopause Society. The 2020 genitourinary syndrome of menopause position statement of The North American Menopause Society. Menopause 2020; 27: 976-992.

15. Ekin $M$, Cengiz $H$, Ayağ $M$, et al. Effects of the levonorgestrel-releasing intrauterine system on urinary symptoms in patients with adenomyosis. Eur J Obstet Gynecol Reprod Biol 2013; 170: 517-520.

16. The 2017 hormone therapy position statement of The North American Menopause Society. Menopause 2017; 24: 728-753.

17. ACOG Committee Opinion No. 659: The Use of Vaginal Estrogen in Women With a History of Estrogen-Dependent Breast Cancer. Obstet Gynecol 2016; 127: e93-e96.

18. Le Ray I, dell'Aniello S, Bonnetain F, et al. Local estrogen therapy and risk of breast cancer recurrence among hormone-treated patients: A nested case-control study. Breast Cancer Res Treat 2012; 135: 603-609.

19. Lethaby A, Ayeleke RO, Roberts $\mathrm{H}$. Local oestrogen for vaginal atrophy in postmenopausal women. Cochrane Database Syst Rev 2016; 2016: CD001500.

20. Rahn DD, Carberry C, Sanses T V, et al. Vaginal estrogen for genitourinary syndrome of menopause. Obstet Gynecol 2014; 124: 1147-1156.

21. Nappi RE, Davis SR. The use of hormone therapy for the maintenance of urogynecological and sexual health post WHI. Climacteric 2012; 15: 267-274. 
22. Bhatia NN, Bergman A, Karram MM. Effects of estrogen on urethral function in women with urinary incontinence. Am J Obstet Gynecol 1989; 160: 176-181.

23. Management of symptomatic vulvovaginal atrophy: 2013 position statement of The North American Menopause Society. Menopause 2013; 20: 888-902; quiz 903-904

24. Naessen T, Rodriguez-Macias K. Endometrial thickness and uterine diameter not affected by ultralow doses of 17beta-estradiol in elderly women. Am J Obstet Gynecol 2002; 186: 944-947.

25. Eugster-Hausmann M, Waitzinger J, Lehnick D. Minimized estradiol absorption with ultra-low-dose 10 microg 17beta-estradiol vaginal tablets. Climacteric 2010; 13: 219-227.

26. Cancer CG on HF in B. Type and timing of menopausal hormone therapy and breast cancer risk: individual participant meta-analysis of the worldwide epidemiological evidence. Lancet 2019; 394: 1159-1168.

27. Bygdeman M, Swahn ML. Replens versus dienoestrol cream in the symptomatic treatment of vaginal atrophy in postmenopausal women. Maturitas 1996; 23: 259-263.

28. Stute $P$. Is vaginal hyaluronic acid as effective as vaginal estriol for vaginal dryness relief? Arch Gynecol Obstet 2013; 288: 1199-1201.

29. Edwards D, Panay N. Treating vulvovaginal atrophy/genitourinary syndrome of menopause: how important is vaginal lubricant and moisturizer composition? Climacteric 2016; 19: 151-161.

30. Perino A, Calligaro A, Forlani F, et al. Vulvo-vaginal atrophy: a new treatment modality using thermo-ablative fractional $\mathrm{CO}_{2}$ laser. Maturitas 2015; 80: 296-301.

31. Salvatore S, Nappi RE, Parma M, et al. Sexual function after fractional microablative $\mathrm{CO}_{2}$ laser in women with vulvovaginal atrophy. Climacteric. 2015;18(2):219-225.

32. Lee MS. Treatment of vaginal relaxation syndrome with an erbium: YAG Laser Using $90^{\circ}$ and $360^{\circ}$ scanning scopes: a pilot study \& short-term results. Laser Ther 2014; 23: 129-138.

33. Pitsouni E, Grigoriadis T, Tsiveleka A, et al. Microablative fractional $\mathrm{CO}_{2}$ laser therapy and the genitourinary syndrome of menopause: An observational study. Maturitas 2016; 94: 131-136.

34. Paraiso MFR, Ferrando CA, Sokol ER, et al. A randomized clinical trial comparing vaginal laser therapy to vaginal estrogen therapy in women with genitourinary syndrome of menopause: The VeLVET Trial. Menopause 2020; 27: 50-56.

35. Bhide AA, Khullar V, Swift S, et al. The use of laser in urogynaecology. Int Urogynecol J 2019; 30:683-692.

36. Salvatore S, Athanasiou S, Candiani M. The use of pulsed CO2 lasers for the treatment of vulvovaginal atrophy. Curr Opin Obstet Gynecol 2015; 27: 504-508.

37. Salvatore S, Leone Roberti Maggiore U, Athanasiou S, et al. Histological study on the effects of microablative fractional $\mathrm{CO}_{2}$ laser on atrophic vaginal tissue: an ex vivo study. Menopause 2015; 22: 845-849.

38. Zerbinati N, Serati M, Origoni $M$, et al. Microscopic and ultrastructural modifications of postmenopausal atrophic vaginal mucosa after fractional carbon dioxide laser treatment. Lasers Med Sci 2015; 30: 429436.

39. Cruz VL, Steiner ML, Pompei LM, et al. Randomized, double-blind, placebo-controlled clinical trial for evaluating the efficacy of fractional $\mathrm{CO} 2$ laser compared with topical estriol in the treatment of vaginal atrophy in postmenopausal women. Menopause 2018; 25: 21-28.

40. Flint R, Cardozo L, Grigoriadis T, et al. Rationale and design for fractional microablative $\mathrm{CO} 2$ laser versus photothermal non-ablative erbium: YAC laser for the management of genitourinary syndrome of menopause: a non-inferiority, single-blind randomized controlled trial. Climacteric 2019; 22: 307-311.

41. Naumova I, Castelo-Branco C. Current treatment options for postmenopausal vaginal atrophy. Int J Womens Health 2018; 10: 387-395.

42. Hutchinson-Colas J, Segal S. Genitourinary syndrome of menopause and the use of laser therapy. Maturitas 2015; 82: 342-345.

43. Gottlieb S. Statement from FDA Commissioner on efforts to safeguard women's health from deceptive health claims and significant risks related to devices marketed for use in medical procedures for "vaginal rejuvenation". FDA. 2018. Availale at: https://www.fda.gov/news-events/ press-announcements/statement-fda-commissioner-scott-gottlieb-mdefforts-safeguard-womens-health-deceptive-health-claims.
44. Colonese F, Laganà AS, Colonese E, et al. The pleiotropic effects of vitamin D in gynaecological and obstetric diseases: an overview on a Hot Topic. Biomed Res Int 2015; 2015: 986281.

45. Abban G, Yildirim NB, Jetten AM. Regulation of the vitamin D receptor and cornifin beta expression in vaginal epithelium of the rats through vitamin D3. Eur J Histochem 2008; 52: 107-114.

46. Sousa MS, Peate M, Jarvis S, et al. A clinical guide to the management of genitourinary symptoms in breast cancer survivors on endocrine therapy. Ther Adv Med Oncol 2017; 9: 269-285.

47. Yildirim B, Kaleli B, Düzcan E, et al. The effects of postmenopausal Vitamin D treatment on vaginal atrophy. Maturitas 2004; 49: 334-337.

48. Lee A, Lee MR, Lee HH, et al. Vitamin D proliferates vaginal epithelium through rhoa expression in postmenopausal atrophic vagina tissue. Mol Cells 2017; 40: 677-684.

49. Keshavarzi Z, Janghorban R, Alipour S, et al. The effect of vitamin D and $E$ vaginal suppositories on tamoxifen-induced vaginal atrophy in women with breast cancer. Support Care Cancer 2019; 27: 1325-1334.

50. Zareai $M$. Effect of vitamin $E$ on the vaginal atrophy of postmenopausal women. Value Heal 2014; 17: A750.

51. Franco OH, Chowdhury R, Troup J, et al. Use of plant-based therapies and menopausal symptoms: a systematic review and meta-analysis. JAMA 2016; 315: 2554-2563.

52. Shakeri F, Taavoni S, Goushegir A, et al. Effectiveness of red clover in alleviating menopausal symptoms: a 12-week randomized, controlled trial. Climacteric 2015; 18: 568-573.

53. Bouchard C. Herbal alternatives as substitutes for hormone therapy in urogenital atrophy. Menopause 2008; 15: 12-13.

54. Madhu C, Enki D, Drake MJ, et al. The functional effects of cigarette smoking in women on the lower urinary tract. Urol Int 2015; 95: 478482.

55. Karamanidis D, Tamiolakis D, Koutsougeras G, et al. Cigarette smoking and the degree of maturation of the vaginal squamous epithelium in postmenopausal women. Clin Exp Obstet Gynecol 2001; 28: 274-276.

56. Mercier J, Morin M, Lemieux M-C, et al. Pelvic floor muscles training to reduce symptoms and signs of vulvovaginal atrophy. Menopause 2016 23: $816-820$

57. Dumoulin C, Hay-Smith EJC, MacHabée-Séguin G. Pelvic floor muscle training versus no treatment, or inactive control treatments, for urinary incontinence in women. Cochrane Database Syst Rev 2018; 10 CD005654.

58. Ghaderi F, Oskouei AE. Physiotherapy for women with stress urinary incontinence: a review article. J Phys Ther Sci 2014; 26: 1493-1499.

59. Al-Saqi SH, Uvnäs-Moberg K, Jonasson AF. Intravaginally applied oxytocin improves post-menopausal vaginal atrophy. Post Reprod Heal 2015; 21: 88-97.

60. Al-Saqi SH, Jonasson AF, Naessén T, et al. Oxytocin improves cytological and histological profiles of vaginal atrophy in postmenopausal women. Post Reprod Heal 2016; 22: 25-33.

61. Torky HA, Taha A, Marie H, et al. Role of topical oxytocin in improving vaginal atrophy in postmenopausal women: a randomized, controlled trial. Climacteric 2018; 21: 174-178.

62. Zohrabi I, Abedi P, Ansari S, et al. The effect of oxytocin vaginal gel on vaginal atrophy in postmenopausal women: A randomized controlled trial. BMC Womens Health 2020; 20: 108.

63. Kallak TK, Uvnäs-Moberg K. Oxytocin stimulates cell proliferation in vaginal cell line Vk2E6E7. Post Reprod Heal 2017; 23: 6-12.

64. Pięta W, Smolarczyk R. Vaginal dehydroepiandrosterone compared to other methods of treating vaginal and vulvar atrophy associated with menopause. Menopause Rev 2020; 19: 195-199.

65. Labrie F, Bélanger A, Pelletier G, et al. Science of intracrinology in postmenopausal women. Menopause 2017; 24: 702-712.

66. Archer DF, Labrie F, Bouchard C, et al. Treatment of pain at sexual activity (dyspareunia) with intravaginal dehydroepiandrosterone (prasterone) Menopause 2015; 22: 950-963.

67. Labrie F, Archer DF, Koltun W, et al. Efficacy of intravaginal dehydroepiandrosterone (DHEA) on moderate to severe dyspareunia and vaginal dryness, symptoms of vulvovaginal atrophy, and of the genitourinary syndrome of menopause. Menopause 2016; 23: 243-256.

68. Labrie F, Archer DF, Bouchard C, et al. Prasterone has parallel beneficial effects on the main symptoms of vulvovaginal atrophy: 52-week openlabel study. Maturitas 2015; 81: 46-56. 
69. Martel C, Labrie F, Archer DF, et al. Serum steroid concentrations remain within normal postmenopausal values in women receiving daily $6.5 \mathrm{mg}$ intravaginal prasterone for 12 weeks. J Steroid Biochem Mol Biol 2016; 159: 142-153.

70. Portman DJ, Labrie F, Archer DF, et al. Lack of effect of intravaginal dehydroepiandrosterone (DHEA, prasterone) on the endometrium in postmenopausal women. Menopause 2015; 22: 1289-1295.

71. Mitlak BH, Cohen FJ. Selective estrogen receptor modulators: a look ahead. Springer, Berlin, 1999, 653-663.

72. Portman DJ, Bachmann GA, Simon JA. Ospemifene, a novel selective estrogen receptor modulator for treating dyspareunia associated with postmenopausal vulvar and vaginal atrophy. Menopause 2013; 20 : 623-630.

73. Bachmann GA, Komi JO. Ospemifene effectively treats vulvovaginal at rophy in postmenopausal women: Results from a pivotal phase 3 study. Menopause 2010; 17: 480-486.

74. Simon JA, Lin VH, Radovich C, et al. One-year long-term safety extension study of ospemifene for the treatment of vulvar and vaginal atrophy in postmenopausal women with a uterus. Menopause 2013; 20: 418-427. 